\title{
Assessment of mHealth Solutions Applied to Fall Detection for the Elderly
}

\author{
Fellipe Soares de OLIVEIRA ${ }^{\text {a }}$, Camila Carvalho da SILVA ${ }^{\text {a }}$, Talita Santos \\ PINHEIRO $^{\text {a }}$, Larissa Mayumi YOKOI ${ }^{\mathrm{a}}$, Pablo Deoclecia dos SANTOS ${ }^{\mathrm{a}}$, \\ Harki TANAKA ${ }^{\text {a }}$, Priscyla Waleska SIMÕES ${ }^{\mathrm{a} 1}$ \\ ${ }^{a}$ Engineering, Modeling and Applied Social Sciences Center (CECS), Biomedical \\ Engineering Graduate Program (PPGEBM), \\ Universidade Federal do ABC (UFABC), Alameda da Universidade s/n, 09606-045 \\ São Bernardo do Campo, SP, Brazil
}

\begin{abstract}
Mobile Health has been increasingly present in healthcare due to the wide availability of applications for smartphones, however, robust assessment methods must be considered, seeking to provide evidence for clinical practice and mHealth solutions. This research presents the assessment of applications aimed at detecting and preventing falls for the elderly, available for Android and IOS, through the Mobile App Rating Scale. Based on the results presented, it can be concluded that the fall detection and prevention applications for the elderly available for Android and IOS showed good quality after rigorous evaluation.
\end{abstract}

Keywords. Digital Health. Mobile Health. Prevention. IoT. Falls. Assessment.

\section{Introduction}

According to the World Health Organization (WHO), falls represent the second leading cause of accidental or unintentional deaths in the world (traffic accidents are the main cause in this group), resulting in approximately 650,000 deaths, and, of these, $80 \%$ in low-and middle-income countries. In this context, individuals over 60 years of age are the most susceptible to fatal falls (424,000 deaths), suggesting that falls for the elderly have become a public health problem[16; 17].

While many falls are not fatal, approximately 38 million of those that occur annually are severe enough to result in hospitalization. The costs of injuries related to falls are substantial, as, among the population of 65 years old or over, the average cost per injury can range from US $\$ 1,049.00$ to US $\$ 3,611.00$ in countries such as Australia and Finland, respectively[16].

Due to the increased use of the internet through smartphones and wearable devices (such as smart watches and bracelets), monitoring strategies are becoming increasingly popular through alerts, for example, in addition to contributing to training actions related to the treatment and prevention of diseases, and improving access to health services, clinical diagnosis and treatment adherence[6].

${ }^{1}$ Corresponding Author: Prof. Dr. Priscyla Waleska Simões, Universidade Federal do ABC, Engineering, Modeling and Applied Social Sciences Center (CECS), Biomedical Engineering Graduate Program (PPGEBM); Alameda da Universidade s/n, 09606-045 São Bernardo do Campo, SP, Brazil; E-mail: pritsimoes@gmail.com. 
In this context, mHealth solutions have been developed for the detection and prevention of falls, through research using cell phone sensors (gyroscope and accelerometer), Machine Learning, among other technologies[10; 13$]$.

Despite mHealth's advances[11] and the development of applications for the detection and prevention of falls aimed at the elderly population in recent years, such solutions rarely present a scientific mHeatlh assessment, and when assessment is performed, the instruments used are not specific to mHealth[7]. Therefore, there are gaps to be clarified for a broader adoption in clinical practice and based on scientific evidence[1].

Thus, the aim of this paper is to answer the question: Are the free applications currently available for the Android and IOS operating systems and aimed at detecting and preventing falls for the elderly, having good quality after rigorous scientific mHealth evaluation? Thus, the objective is to present an evaluation of mHealth solutions for the detection and prevention of falls for the elderly.

\section{Methods}

Study on the assessment of applications for the detection and prevention of falls for the elderly, which started with the search strategy carried out by 2 researchers independently, being followed by the description of the applications, categorization and analysis of features. The last step presented the assessment of applications by MARS, ending with the statistical analysis.

The search strategy was carried out through a search in the digital distribution service of Google (Google Play) and Apple (App Store), as they are the platforms associated with the most used operating systems in the world for mobile applications (Android and iOS, respectively).

The assessment was conducted in the 2nd semester of 2020 and 1st semester of 2021 and used the following keywords and synonyms: "Fall" and "Elderly". Applications made available free of charge on the digital distribution service offered by Google (Google Play) and by Apple (App Store), and that presented the description provided by the developer, to identify the purpose of the study and the target population, were included in the study.

Applications with functionality presented in Portuguese, English and Spanish were also included, and applications with inoperable features, paid items (and not provided by the developer), and those that do not directly presented information on the detection and prevention of falls were excluded. The application screening and eligibility process is shown in figure 1.

In evaluating the applications, a scale focused on mHealth was used, the Mobile Application Rating Scale (MARS) [5], which is a questionnaire that measures the quality of health applications based on 23 questions organized into 4 objective categories (engagement, functionality, aesthetics and information quality) and 1 subjective category. Each question is rated on a 5-point scale (1-inadequate, 2-poor, 3-acceptable, 4-good, 5excellent)[14].

The assessment of the apps was presented by the average, used as a measure of central tendency, and the standard deviation to quantify its variability. To identify the correlation between the categories (A, B, C, D, and E subjective) and the Final Score, the Pearson's correlation coefficient test was used. For all statistical analysis, a 
significance level of 5\% was considered. All statistical analysis were performed using Jamovi software (The Jamovi Project, Sydney, Australia) version 1.6.3.

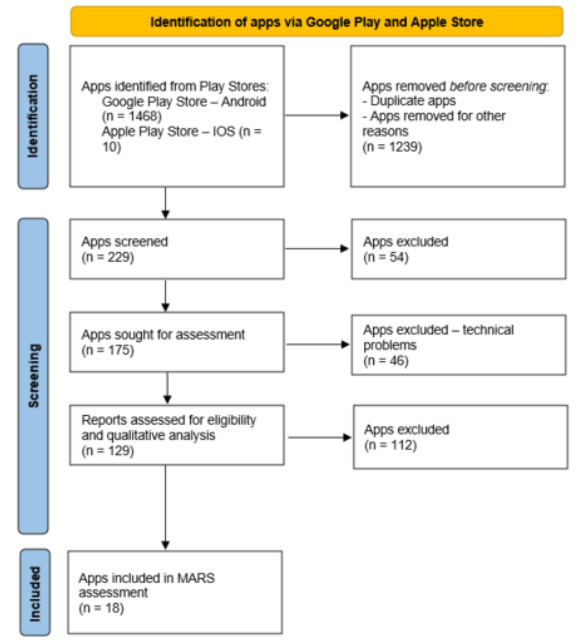

Figure 1. Flowchart of app selection

\section{Results and Discussion}

The assessment resulted in 18 apps that were rated by the MARS scale. The results indicated a positive correlation between the Final Score and Engagement $(\mathrm{r}=0.872$; $\left.\mathrm{R}^{2}=76.0 \% ; \mathrm{p}<0.001\right)$; Functionality $\left(\mathrm{r}=0.498 ; \mathrm{R}^{2}=24.8 \% ; \mathrm{p}=0.042\right)$; Aesthetics $(\mathrm{r}=0.909$; $\left.\mathrm{R}^{2}=82.6 \% ; \mathrm{p}<0.001\right)$; Information $\left(\mathrm{r}=0.838 ; \mathrm{R}^{2}=70.2 \% ; \mathrm{p}<0.001\right)$; Subjective $(\mathrm{r}=0.953$; $\left.\mathrm{R}^{2}=90.8 \% ; \mathrm{p}<0.001\right)$.

Table 1 presents the main features of the apps. It is important to note that 4 applications were compatible with wearables (smartwatch), that is, the development of applications for wearable devices for monitoring falls is still rarely used. In the analysis of the use of sensors, applications aimed at health education actions for patients did not present sensors, however, all applications that detect falls use the accelerometer sensor, which is the main resource used to detect the vibrations associated with falls.

In addition to these features, some applications make use of the gyroscope sensor that together with the accelerometer sensor can improve the accuracy of the fall detection. The use of the GPS sensor by some applications contributes to the user's physical monitoring, thus, regardless of geographic location, GPS makes it possible to record the place where the fall occurs, which can be used as an alert to emergency contacts and immediate help.

The assessment was performed using the MARS scale shown in Table 2, which is organized in descending order of the Final Score, with general average ranging from 4.75 $( \pm 0.25)$ to $1.86( \pm 0.69)$.

MARS has been widely used since 2015[9], and to present, the literature has more than 100 related publications (www.pubmed.com). This instrument has become widespread as can be seen in a review of urinary incontinence management applications[2]. Both studies[2;9] suggest the importance of using scales such as MARS in the assessment stage, seeking to improve mHealth solutions. 
Table 1. Sample characteristics

\begin{tabular}{|c|c|c|c|c|c|c|c|c|c|c|}
\hline APP & Link & Language & os & COMPATIBILITY & FREEWARE & CATEGORY & SUBCATEGORY & ACCESSORIES & SENSORS & ML \\
\hline 1 & $\begin{array}{l}\text { hitps:///apps.appleccom/br/app falllsatety-pro- } \\
\text { safety-alerts/id770864283 }\end{array}$ & English & Android/IOS & $\begin{array}{l}\text { Requires Android } 5.0 \text { or } \\
\text { higher / iOS } 12.0 \text { and } \\
\text { watchoS } 5.0 \text { or higher. }\end{array}$ & No & Patient Centered & Health Tracking & $\begin{array}{l}\text { Smartphone / } \\
\text { Smartwatch }\end{array}$ & $\begin{array}{l}\text { Accelerometer/ } \\
\text { Gyroscope / GPS }\end{array}$ & Yes \\
\hline 2 & 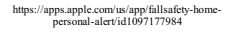 & English & IOS & $\begin{array}{l}\text { iOS } 9.0 \text { and watchoS } \\
2.0 \text { or higher }\end{array}$ & No & Patient Centered & Health Tracking & $\begin{array}{l}\text { Smartphone/ } \\
\text { Smartwatch }\end{array}$ & $\begin{array}{l}\text { Accelerometer/ } \\
\text { Gyroscope/GPS }\end{array}$ & Yes \\
\hline 3 & 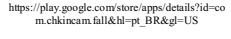 & English & Android & Android 5.0 or higher & No & Patient Centered & Health Tracking & $\begin{array}{l}\text { Smartphone/ } \\
\text { Smartwatch }\end{array}$ & $\begin{array}{l}\text { Accelerometer/ } \\
\text { Gyroscope / GPS }\end{array}$ & - \\
\hline 4 & 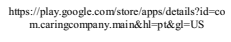 & English & Android / IOS & $\begin{array}{l}\text { Android } 5.0 \text { or higher } / \\
\text { iOS } 13.0 \text { or higher }\end{array}$ & No & Patient Centered & Health Tracking & Smartphone & Accelerometer/GPS & Yes \\
\hline 5 & $\begin{array}{c}\text { https://play.google.com/store/apps/details? } \\
\text { id=com.tidyware.workersafety.pro \&hl=en_US } \\
\text { \&gle=US }\end{array}$ & English & Android / IOS & $\begin{array}{l}\text { Android } 5.0 \text { or higher / } \\
\text { iOS } 13.0 \text { or higher }\end{array}$ & No & Patient Centered & Health Tracking & Smartphone & $\begin{array}{l}\text { Accelerometer/ } \\
\text { Gyroscope / GPS }\end{array}$ & - \\
\hline 6 & 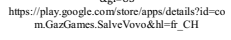 & Portuguese & Android & Android 2.3 or higher & Yes & Educational & Patient Education & Smartphone & No need & No \\
\hline 7 & 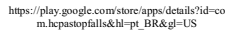 & English & Android / IOS & $\begin{array}{l}\text { Android } 5.0 \text { or higher } / \\
\text { iOS } 10.3 \text { or higher }\end{array}$ & Yes & Educational & Patient Education & Smartphone & No need & No \\
\hline 8 & $\begin{array}{l}\text { hitps://play.googlec.con/store/apps/details?id=co } \\
\text { m.healthappy.seizario2\&hl=pt_BR\&gl =US }\end{array}$ & English & Android & Android 5.0 or higher & Yes & Patient Centered & Health Tracking & Smartphone & Accelerometer/GPS & - \\
\hline 9 & 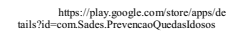 & Portuguese & Android & Android 4.4 or higher & Yes & Educational & Patient Education & Smartphone & No need & No \\
\hline 10 & $\begin{array}{l}\text { https://play.googlec.com/store/apps/details?id=co } \\
\text { m.Jump.FallsPrevention }\end{array}$ & English & Android / IOS & $\begin{array}{l}\text { Android } 4.1 \text { or higher/ } \\
\text { iOS } 10.0 \text { or higher }\end{array}$ & Yes & Educational & Patient Education & Smartphone & $\begin{array}{l}\text { Accelerometer/ } \\
\text { Gyroscope }\end{array}$ & No \\
\hline 11 & $\begin{array}{l}\text { https://play.google.con/store/apps/detetils?'id=co } \\
\text { m.fallddetection }\end{array}$ & English & Android & Android 4.1 or higher & Yes & Patient Centered & Health Tracking & Smartphone & Accelerometer & Yes \\
\hline 12 & $\begin{array}{l}\text { https:://play.googlec.courstore/apps/details?id=co } \\
\text { m.HRscape, grandzangel }\end{array}$ & English & Android & Android 4.1 or higher & Yes & Patient Centered & Health Tracking & Smartphone & Accelerometer & 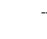 \\
\hline 13 & $\begin{array}{l}\text { https://apps.applecom/usiapp/alarm- } \\
\text { free id } 284728433\end{array}$ & English & IOS & iOS 12.0 or higher & Yes & Patient Centered & Health Tracking & Smartphone & $\begin{array}{l}\text { Accelerometer/ } \\
\text { Gyroscope / GPS }\end{array}$ & No \\
\hline 14 & 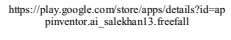 & English & Android & Android 1.5 or higher & Yes & Patient Centered & Health Tracking & $\begin{array}{l}\text { Smartphone/ } \\
\text { Smartwatch }\end{array}$ & $\begin{array}{l}\text { Accelerometer/ } \\
\text { Gyroscope }\end{array}$ & \\
\hline 15 & $\begin{array}{l}\text { https://apps.apple.com/ptrapp/care24idid 1 152041 } \\
\qquad 883\end{array}$ & English & IOS & iOS 12.0 or higher & Yes & Patient Centered & Health Tracking & Smartphone & Accelerometer/GPS & No \\
\hline 16 & 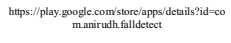 & English & Android & Android 7.0 or higher & Yes & Patient Centered & Health Tracking & Smartphone & $\begin{array}{l}\text { Accelerometer/ } \\
\text { Gyroscope / GPS }\end{array}$ & Yes \\
\hline 17 & 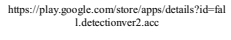 & English & Android & Android 5.1 or higher & Yes & Patient Centered & Health Tracking & Smartphone & $\begin{array}{l}\text { Accelerometer/ } \\
\text { Gyroscope }\end{array}$ & \\
\hline 18 & $\begin{array}{l}\text { https://play.google.con/store/apps/details?id=co } \\
\text { m.sjinnovation gameoffalls.live }\end{array}$ & English & Android & Android 5.0 or higher & Yes & Patient Centered & Patient Education & Smartphone & - & No \\
\hline
\end{tabular}

OS - Operating System / ML - Machine Learning

Considering the MARS domains, category A, which covers issues related to engagement, was the category with the lowest overall average in our study (3.43 \pm 0.93$)$. Engagement strategies such as gamification were not relevant to the context of falling for the elderly, however, such techniques have shown good results in mHealth solutions related to Nutrition in children and adolescents[12].

In category B related to features, there was a higher overall average ( $4.24 \pm 0.45)$, due to the easy use of the applications, simple and objective design, and few gaps related to navigation.

In category $\mathrm{C}(3.58 \pm 0.36)$, which evaluates the proportion of components in the interface, graphic quality of icons and aesthetic appeal, for example, the average was possibly influenced by applications that did not present a visually attractive interface (such as buttons in irregular positions).

In category $\mathrm{D}(3.60 \pm 0.92)$, the relevance of the information and the presence of illustrations were observed to facilitate navigation and understanding of the application. No apps were scientifically evaluated, and the patient-centric apps did not contain information relevant to fall prevention. Educational applications had a higher Final Average because their assessment took this category into consideration.

Some applications had manual pushbuttons and alerts to external contacts after the fall was detected, however, as the fall can immobilize the patient, this feature can influence the alert, help and assistance. In this context, in some studies[3;4;18], the accelerometer sensor on a wearable/smartphone is the main feature used in fall detection as it easily identifies the vibrations associated with the fall. 
Table 2. MARS scores

\begin{tabular}{|c|c|c|c|c|c|c|c|}
\hline App & Link & A & B & C & D & Average & $E$ \\
\hline 1 & $\begin{array}{l}\text { https://apps.apple.com/br/app/fallsafety-pro- } \\
\text { safety-alerts/id870864283 }\end{array}$ & $5.00 \pm 0.00$ & $4.50 \pm 0.50$ & $5.00 \pm 0.00$ & $4.50 \pm 0.76$ & $4.75 \pm 0.25$ & $5.00 \pm 0.00$ \\
\hline 2 & $\begin{array}{l}\text { https://apps.apple.com/us/app/fallsafety- } \\
\text { home-personal-alert/id1097177984 }\end{array}$ & $5.00 \pm 0.00$ & $4.50 \pm 0.50$ & $5.00 \pm 0.00$ & $4.33 \pm 0.75$ & $4.71 \pm 0.30$ & $5.00 \pm 0.00$ \\
\hline 3 & $\begin{array}{l}\text { https://play.google.com/store/apps/details?id } \\
\text { =com.chkincam.fall\&hl=pt_BR\&gl=US }\end{array}$ & $4.40 \pm 0.49$ & $5.00 \pm 0.00$ & $5.00 \pm 0.00$ & $4.33 \pm 1.11$ & $4.68 \pm 0.32$ & $5.00 \pm 0.00$ \\
\hline 4 & $\begin{array}{l}\text { https://play.google.com/store/apps/details?id } \\
=\text { com.caringcompany.main\&hl=pt\&gl=US }\end{array}$ & $4.40 \pm 0.80$ & $5.00 \pm 0.00$ & $4.67 \pm 0.47$ & $4.60 \pm 0.80$ & $4.67 \pm 0.22$ & $5.00 \pm 0.00$ \\
\hline 5 & $\begin{array}{l}\text { https://play.google.com/store/apps/details?id } \\
=\text { com.tidyware.workersafety.pro\&hl=en_U } \\
\text { S\&gl=US }\end{array}$ & $4.80 \pm 0.45$ & $4.25 \pm 0.83$ & $5.00 \pm 0.00$ & $4.00 \pm 0.82$ & $4.51 \pm 0.40$ & $3.25 \pm 0.43$ \\
\hline 6 & $\begin{array}{l}\text { https://play.google.com/store/apps/details?id } \\
=\text { com.GazGames.SalveVovo\&hl=fr_CH }\end{array}$ & $4.00 \pm 1.26$ & $4.75 \pm 0.43$ & $5.67 \pm 0.47$ & $4.17 \pm 0.69$ & $4.40 \pm 0.32$ & $3.50 \pm 1.12$ \\
\hline 7 & $\begin{array}{l}\text { https://play.google.com/store/apps/details?id } \\
=\text { com.hcpastopfalls\&hl=pt_BR\&gl=US }\end{array}$ & $3.40 \pm 1.96$ & $5.00 \pm 0.00$ & $4.33 \pm 0.47$ & $4.67 \pm 0.75$ & $4.35 \pm 0.60$ & $4.50 \pm 0.87$ \\
\hline 8 & $\begin{array}{l}\text { https://play.google.com/store/apps/details?id } \\
=\text { com.healthappy.seizario2\&hl=pt_BR\&gl= } \\
\text { US }\end{array}$ & $4.60 \pm 0.49$ & $4.75 \pm 0.43$ & $3.67 \pm 0.94$ & $4.33 \pm 0.75$ & $4.34 \pm 0.42$ & $4.50 \pm 0.50$ \\
\hline 9 & $\begin{array}{l}\text { https://play.google.com/store/apps/details?id } \\
\text { =com.Sades.PrevencaoQuedasIdosos }\end{array}$ & $3.20 \pm 1.17$ & $5.00 \pm 0.00$ & $4.00 \pm 0.00$ & $4.00 \pm 1.00$ & $4.05 \pm 0.64$ & $2.00 \pm 1.00$ \\
\hline 10 & $\begin{array}{l}\text { https://play.google.com/store/apps/details?id } \\
\text { =com.Jump.FallsPrevention }\end{array}$ & $4.20 \pm 1.17$ & $3.50 \pm 0.50$ & $3.67 \pm 0.47$ & $4.50 \pm 0.76$ & $3.97 \pm 0.40$ & $2.50 \pm 0.50$ \\
\hline 11 & $\begin{array}{l}\text { https://play.google.com/store/apps/details?ic } \\
\text { =com.fall.detection }\end{array}$ & $2.80 \pm 1.33$ & $5.00 \pm 0.00$ & $3.67 \pm 0.47$ & $3.83 \pm 0.90$ & $3.83 \pm 0.78$ & $2.50 \pm 1.66$ \\
\hline 12 & $\begin{array}{l}\text { https://play.google.com/store/apps/details?ic } \\
\text { =com.HRscape.grandzangel }\end{array}$ & $4.20 \pm 0.75$ & $3.75 \pm 0.43$ & $2.00 \pm 0.00$ & $4.20 \pm 0.75$ & $3.54 \pm 0.91$ & $2.00 \pm 0.75$ \\
\hline 13 & $\begin{array}{l}\text { https://apps.apple.com/us/app/alarm- } \\
\text { free/id284728433 }\end{array}$ & $1.80 \pm 0.75$ & $4.75 \pm 0.43$ & $2.67 \pm 0.94$ & $2.50 \pm 1.26$ & $2.93 \pm 1.10$ & $1.25 \pm 0.43$ \\
\hline 14 & $\begin{array}{l}\text { https://play.google.com/store/apps/details?id } \\
\text { =appinventor.ai_salekhan13.freefall }\end{array}$ & $2.00 \pm 1.26$ & $5.00 \pm 0.00$ & $1.67 \pm 0.94$ & $2.75 \pm 1.79$ & $2.85 \pm 1.30$ & $1.25 \pm 0.43$ \\
\hline 15 & $\begin{array}{l}\text { https://apps.apple.com/pt/app/care24/id1 } 152 \\
041483\end{array}$ & $2.20 \pm 0.98$ & $3.75 \pm 0.83$ & $3.00 \pm 0.00$ & $1.80 \pm 0.98$ & $2.69 \pm 0.75$ & $1.00 \pm 0.00$ \\
\hline 16 & $\begin{array}{l}\text { https://play.google.com/store/apps/details?id } \\
\text { =com.anirudh.falldetect }\end{array}$ & $2.00 \pm 0.89$ & $4.00 \pm 1.22$ & $1.33 \pm 0.47$ & $2.25 \pm 1.09$ & $2.40 \pm 0.98$ & $1.25 \pm 0.43$ \\
\hline 17 & $\begin{array}{c}\text { https://play.google.com/store/apps/details?id } \\
\text { =fall.detectionver2.acc }\end{array}$ & $2.40 \pm 0.80$ & $2.50 \pm 1.50$ & $2.00 \pm 0.82$ & $2.17 \pm 1.21$ & $2.27 \pm 0.20$ & $1.25 \pm 0.43$ \\
\hline \multirow[t]{2}{*}{18} & $\begin{array}{l}\text { https://play.google.com/store/apps/details?id } \\
\text { =com.sjinnovation. gameoffalls. live }\end{array}$ & $1.40 \pm 0.49$ & $1.25 \pm 0.43$ & $3.00 \pm 0.00$ & $1.80 \pm 0.40$ & $1.86 \pm 0.69$ & $1.00 \pm 0.00$ \\
\hline & Final average & $3.43 \pm 0.93$ & $4.24 \pm 0.45$ & $3.58 \pm 0.36$ & $3.60 \pm 0.92$ & $3.71 \pm 0.59$ & $2.88 \pm 0.47$ \\
\hline
\end{tabular}

A - engagement; B - functionality; C - aesthetics; D - information; Final - final average, E - subjective

Also, optimization methods for fall detection algorithms have been researched in recent years, through a previously defined threshold[15; 19], and Machine Learning[8; $10]$, despite the fact that our sample has considered this last method little.

\section{Conclusion}

Based on the results presented, it can be concluded that the fall detection and prevention applications available for Android and IOS have good quality after a rigorous assessment carried out via MARS scale. A possible limitation was that many elderly people do not use the smartphone as often as young people, and this can impact the number of applications developed for this purpose.

It is recommended that future research address the development of assessment instruments which are adapted to a context where mHealth solutions are involved. Thus, we would suggest a new method of assessment or the continuation of studies for the development of an app that detect and prevents falls, aimed at the elderly population stemming from the gaps shown in the article. 


\section{Acknowledgments}

This study was financed in part by the Coordenação de Aperfeiçoamento de Pessoal de Nível Superior - Brazil (CAPES) - Finance Code 001, and Conselho Nacional de Desenvolvimento Científico e Tecnológico (CNPq) - Brazil.

\section{References}

[1] Byambasuren O, Sanders S, Beller E, Glasziou P. Prescribable mHealth apps identified from an overview of systematic reviews. NPJ Digit Med 2018; 1: 12.

[2] Dantas LO, Carvalho C, B.L.d.J. Santos, C.H.J. Ferreira, K. Bø, and P. Driusso, Mobile health technologies for the management of urinary incontinence: A systematic review of online stores in Brazil. Braz J Phys Ther. Jul-Aug 2021;25(4):387-395. doi: 10.1016/j.bjpt.2021.01.001.

[3] Er PV, Tan KK. 4 - Wearable solution for robust fall detection. In: Assistive Technology for the Elderly, N.K. Suryadevara and S.C. Mukhopadhyay, eds., Academic Press, 2020, pp. 81-105.

[4] Fakhrulddin SS, Gharghan KS. An Autonomous Wireless Health Monitoring System Based on Heartbeat and Accelerometer Sensors. J. Sens. Actuator Netw. 2019; 8(3): 39. https://doi.org/10.3390/jsan8030039.

[5] Gong E, Zhang Z, Jin X, Liu Y, Zhong L, et al. Quality, Functionality, and Features of Chinese Mobile Apps for Diabetes Self-Management: Systematic Search and Evaluation of Mobile Apps JMIR Mhealth Uhealth 2020; 8: e14836.

[6] Gurman TA, Rubin SE, Roess AA. Effectiveness of mHealth behavior change communication interventions in developing countries: a systematic review of the literature. J Health Commun 2012; 17 Suppl 1: 82-104.

[7] Hsieh KL, Fanning JT, Rogers WA, Wood TA, Sosnoff JJ. A Fall Risk mHealth App for Older Adults: Development and Usability Study. JMIR aging 2018; 1: e11569-e11569.

[8] Jahanjoo A, Tahan MN, Rashti MJ. Accurate fall detection using 3-axis accelerometer sensor and MLF algorithm. In: 2017 3rd International Conference on Pattern Recognition and Image Analysis (IPRIA), 2017, pp. 90-95.

[9] Mani M, Kavanagh DJ, Hides L, Stoyanov SR. Review and Evaluation of Mindfulness-Based iPhone Apps. JMIR Mhealth Uhealth 2015; 3: e82.

[10] Nooruddin S, Milon Islam M, Sharna FA. An IoT based device-type invariant fall detection system. Internet of Things 2020; 9: 100130.

[11] Sahlab N, Jazdi N. AI-Based Elderly Assistance Systems. Stud Health Technol Inform 2020; 273 : 163 169.

[12] Schoeppe S, Alley S, Rebar AL, Hayman M, Bray NA, et al. Apps to improve diet, physical activity and sedentary behaviour in children and adolescents: a review of quality, features and behaviour change techniques. International Journal of Behavioral Nutrition and Physical Activity 2017; 14: 83.

[13] Silva J, Gomes D, Sousa I. Technological Solution for Pervasive Fall Risk Assessment. Stud Health Technol Inform 2020; 273: 176-181.

[14] Stoyanov SR, Hides L, Kavanagh DJ, Zelenko O, Tjondronegoro D, Mani M. Mobile App Rating Scale: A New Tool for Assessing the Quality of Health Mobile Apps. JMIR Mhealth Uhealth 2015; 3: e27.

[15] Wang F-T, Chan H.L, Hsu M-H, Lin C-K, Chao P-K, Chang Y-J. Threshold-based fall detection using a hybrid of tri-axial accelerometer and gyroscope. Physiological Measurement 2018; 39: 105002.

[16] World Health Organization. WHO, Falls, in, 2021.

[17] Wu H, Ouyang P. Fall prevalence, time trend and its related risk factors among elderly people in China. Arch Gerontol Geriatr 2017; 73: 294-299.

[18] Yacchirema D, de Puga JS, Palau C, Esteve M. Fall detection system for elderly people using IoT and ensemble machine learning algorithm. Personal and Ubiquitous Computing 2019; 23: 801-817.

[19] Yang L, Ren Y, Zhang W. 3D depth image analysis for indoor fall detection of elderly people. Digital Communications and Networks 2016; 2: 24-34. 